\title{
FUNCTIONAL AND PARAMETRICAL CONTROL OF TEMPERATURE SENSORS WITH THE NI MYRIO MODULE
}

\author{
Kirill Moskalenko a, Kuandyk Omar, Pavel Nekrasov, Andrey Karakozov, Dmitry \\ Boychenko \\ National Research Nuclear University MEPhI (Moscow Engineering Physics Institute), 115409, \\ Moscow, Russia
}

\begin{abstract}
Developed automated setup for functional and parametrical control of temperature sensors is presented. The hardware setup is based on the NI myRIO operated under LabVIEW software. Two ways of communication with the temperature sensor, advantages and disadvantages are described. The experimental results functional and parametrical control for three temperature sensors are presented.
\end{abstract}

\section{Introduction}

The digital temperature sensor is one of the most used elements in different electrical systems [1-4]. It determines the object or environment temperatures with the required accuracy $[5,6]$. The advantages of the sensor with digital interface $[7,8]$ are noise immunity when receiving data and the possibility to connect several devices to a single bus.

Usually the input control of the digital or analog integrated circuit is performed by automated systems or complexes of the functional and parametric control [9-11]. In this paper we describe the system of the parametrical and functional control for temperature sensor DS1624S+ [12].

\section{Device under test}

The temperature sensor DS1624S+ is packaged in PDIP package and consisted of two separate functional blocks: 256B EEPROM and digital temperature sensor. The interaction with the integrated circuit occurs through $\mathrm{I} 2 \mathrm{C}$ protocol. The read and converted temperature is loaded in a 12-bit word. The temperature range is from -55 to +125 degrees Celsius with the accuracy of 0.0625 degrees. Maximum conversion time is $200 \mathrm{~ms}$.

The developed system has to provide the following features:

- a connection and control of up to 4 temperature sensors;

- an independent power supply from $2.7 \mathrm{~V}$ up to $5.5 \mathrm{~V}$ on every integrated circuit;

- measurements of the current consumption with accuracy up to $0.1 \mathrm{uA}$;

- measurements of the output logic levels $\mathrm{V}_{\mathrm{OL}}$ and $\mathrm{V}_{\mathrm{OH}}$;

\footnotetext{
${ }^{a}$ Corresponding author: shaxtyr@yandex.ru
} 
- writing and reading data via serial interface.

\section{Setup requirements}

Hardware part of the system consists of National Instruments (NI) myRIO module and SANWA PC5000 multimeter. Application circuit for the temperature sensor is shown on Figure 1. Power supply, data exchange and output logic levels measurement are implemented on the base of NI myRIO input/output lines [13].

Multimeter is used for current measurement in the power supply circuit and is controlled via RS-232 interface from computer. Custom software was developed in LabVIEW development system.

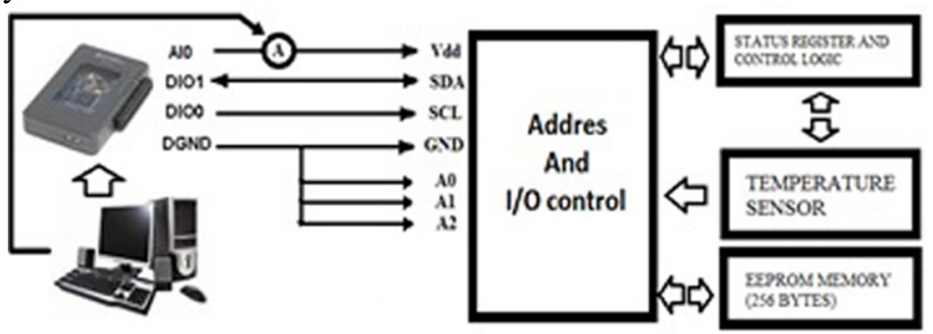

Figure 1. Temperature sensor circuit.

\section{Solution}

Serial interface for data exchange was be implemented to provide the functional control (Figure 2). Each sensor must be connected to a separate bus to exclude the mutual influence of the remaining sensors during testing.

Two ways of communication with the sensor were implemented:

- built-in hardware I2C interface of the myRIO module;

- NI myRIO FPGA $[14,15]$ based implementation of the I2C interface.

\section{Write to DS1624}

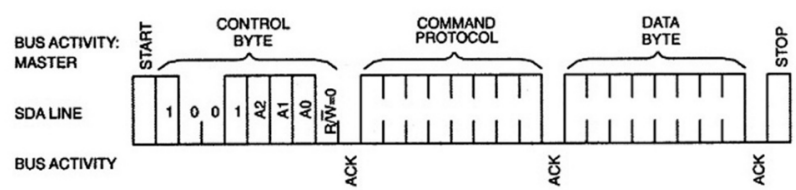

\section{Read from DS1624}

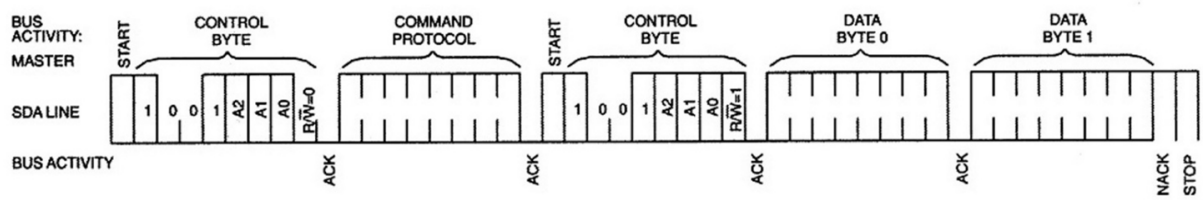

Figure 2. Algorithm of reading and writing to DS1624S + .

The built-in hardware I2C interface module is an advantage of the myRIO system (Figure 3). This virtual instrument implemented on the target machine (the target machine is realized as such construction: a structure "case" is in a loop "while"; a variant of "case" is 
chose by constant "enum") has the capability of single and continuous temperature acquisition, reading/writing data to memory, the ability to write the configuration register and read data from it (Figure 4). The front panel that virtual instrument is shown on Figure 5.

However it allows independent simultaneous connection of only 2 sensors because of a number of ports on myRIO for $\mathrm{I} 2 \mathrm{C}$ is limited.

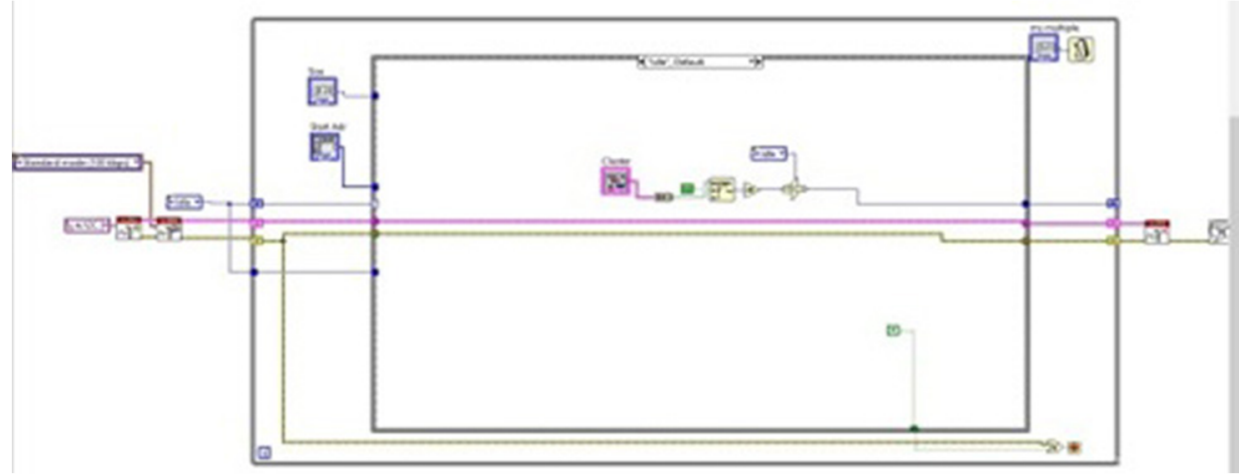

Figure 3. Main state machin.

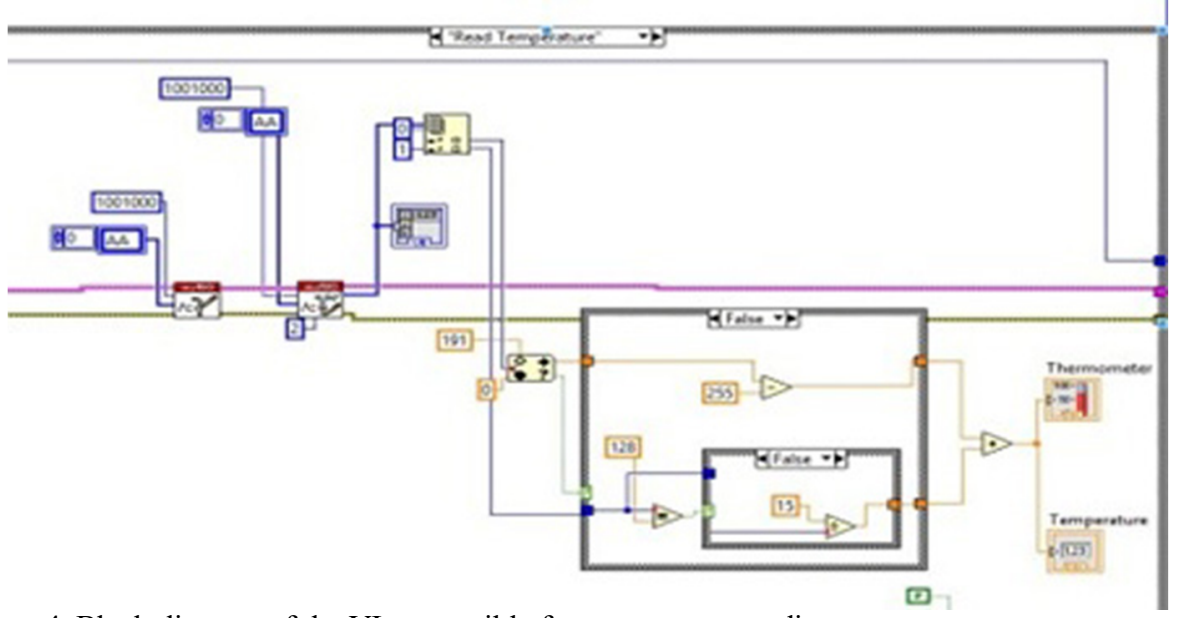

Figure 4. Block diagram of the VI responsible for temperature reading.
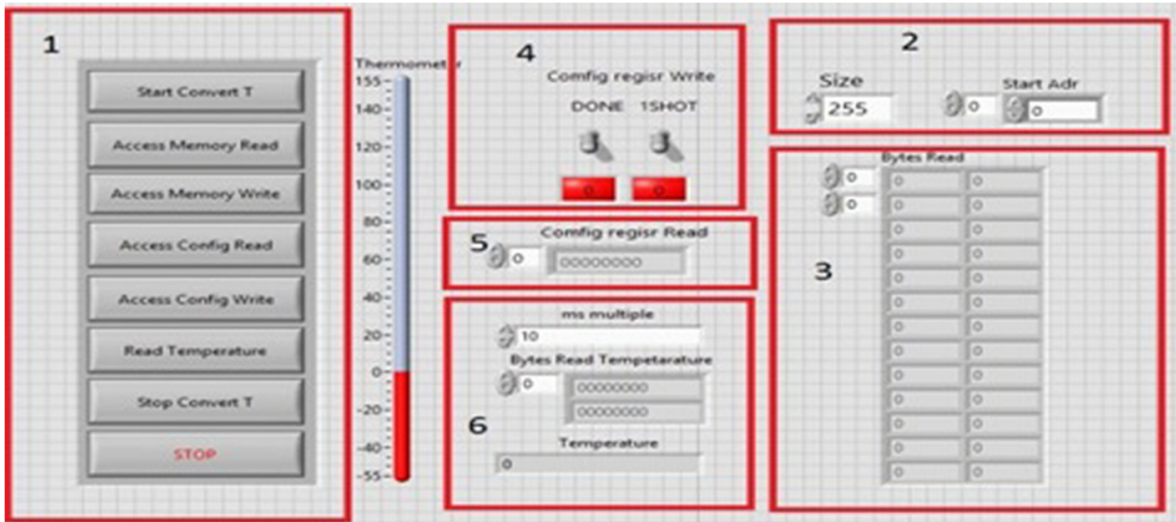

Figure 5. Front panel of the VI: 1-operation select; 2-3-size of the memory to write and to read to; 4configuration register setup; 5-reading settings for the configuration register; 6 -temperature value. 
The other solution for problem was based on FPGA. The possibility to implement about 20 independent digital interfaces $\mathrm{I} 2 \mathrm{C}$ is advantage of that method.

Computer software consists of 2 paths - HOST.vi and FPGA.vi. FPGA implements data exchange via serial interface on low level according to the sensor documentation. Transfer algorithm of the data frame was implemented by using developed hardware reading (Figure 6 ) and writing (Figure 7) subsidiary virtual instruments. These subsidiary virtual instruments provide time characteristics according to the $\mathrm{I} 2 \mathrm{C}$ protocol.

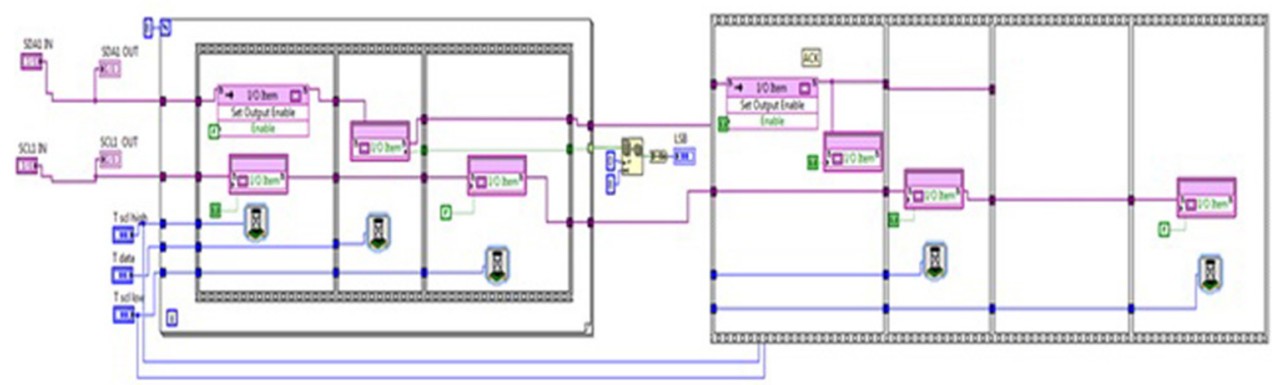

Figure 6. Reading byte VI.

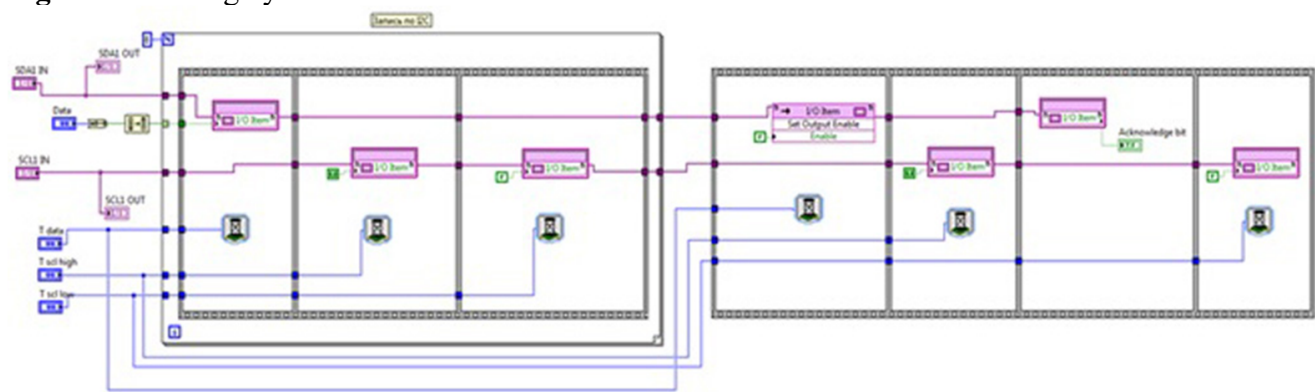

Figure 7. Writing byte VI.

HOST module controls temperature sensor parameters, implements on the high level data protocols (Figure 8). Everyone commands are represented their hexadecimal number according to documentation [5]. During the testing process one of the four operation modes could be chosen: waiting, temperature measurement in degrees Celsius, also reading / writing from / in EEPROM a control command. Information is shown on indicators.

During the regime "a measurement temperature" reset of information is happened and protocol "writing information in DS1624S+" is implemented. First a writing control byte which last bit is " 0 " is realized using under virtual instrument "writing of byte". Then a command which signals about beginning of conversion temperature is written. Later the protocol "reading information from DS1624S+" is realized. Writing a control byte which last bit is "0", is realized using under virtual instruments "writing byte" and "reading byte", then a command which signals about beginning of reading temperature is realized and a control byte which last bit is "1" according to protocols of exchange information.

The measured temperature was written in two bytes: MSB - integer part, and LSB factorial part which four least significant bits are null according to documentation [5]. The front panel of HOST.vi is showed on Figure 9.

A power supply was provided by analog power lines of NI myRIO. They can provide up to $3 \mathrm{~mA}$. This is enough to ensure the sufficient current consumption of the sensor, which peak value should not exceed $1.2 \mathrm{~mA}$. 


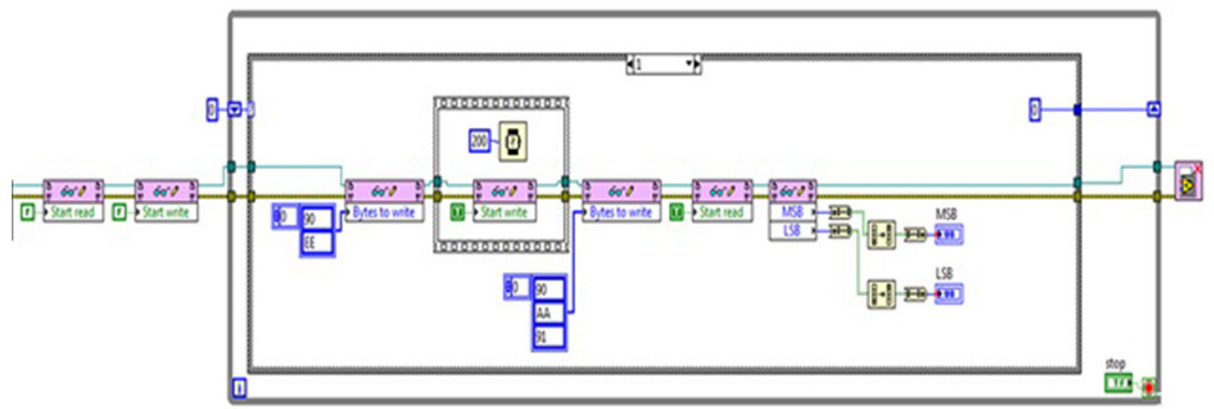

Figure 8. HOST.vi Block-diagram.

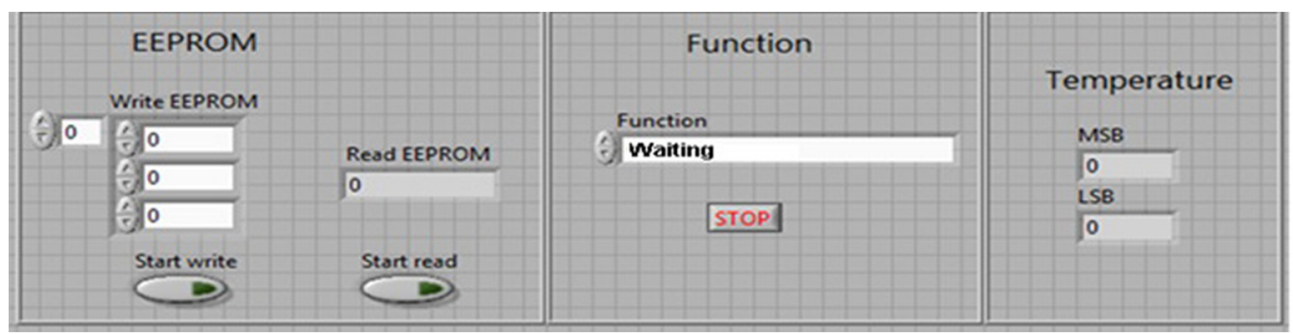

Figure 9. Front panel of the HOST.vi.

\section{Experimental results}

The experimental research including functional and parametrical control of the operation of the temperature sensors under the destabilizing influence was conducted for the DS1624S+ temperature sensor. Only 3 samples were examined. Two samples measured a stabilized environmental temperature, i.e. $21^{\circ} \mathrm{C}$. Third sample measured the temperature of a hot airflow which was $50^{\circ} \mathrm{C} \ldots 60^{\circ} \mathrm{C}$. The plots of measured temperature on experiment time are shown on Figure 10. End of plot line determines fault of the temperature reading from sensor. Internal flash fault happened about $1.4 \cdot 10^{4} \mathrm{sec}$. from the beginning of the experiment. Flash test consisted of sequential writing and reading of reference data. On the fault time it revealed loss of possibility to rewrite the information; it remained the same after the write procedure. Measured sensors' dynamic and standby currents vs time curves are shown on Figure 11 and Figure 12 respectively. 


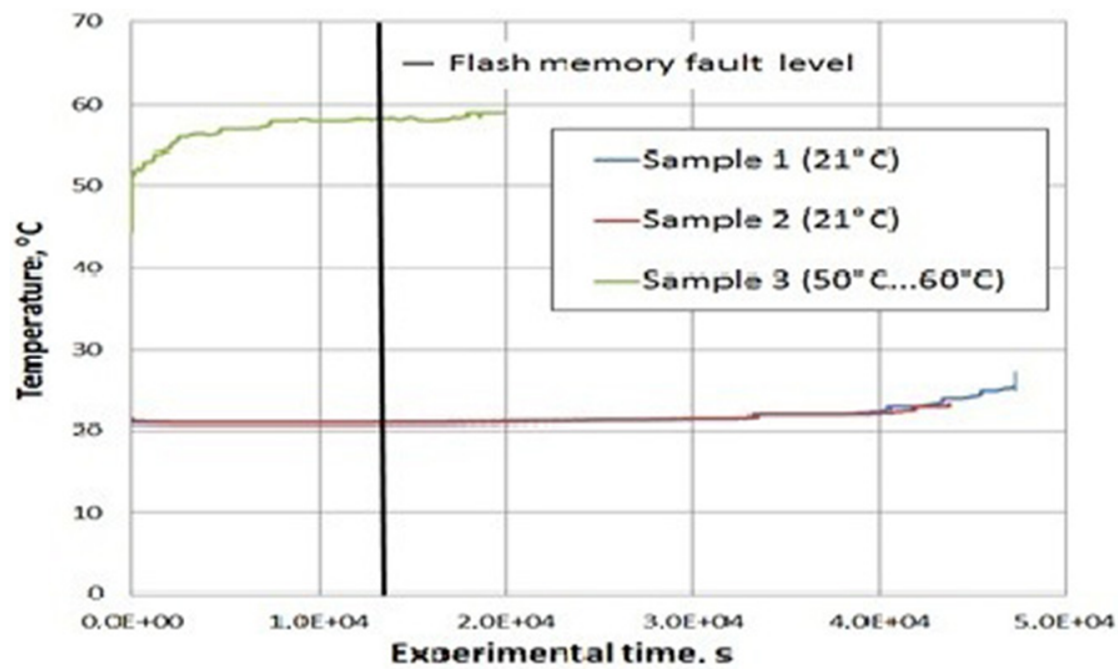

Figure 10. Sensors' temperature vs time.

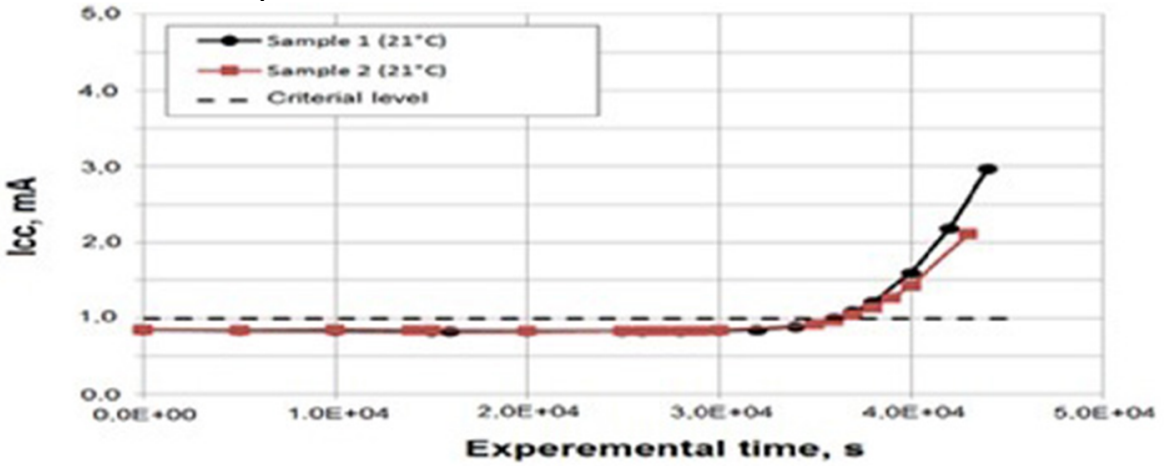

Figure 11. Sensors' dynamic current vs time.

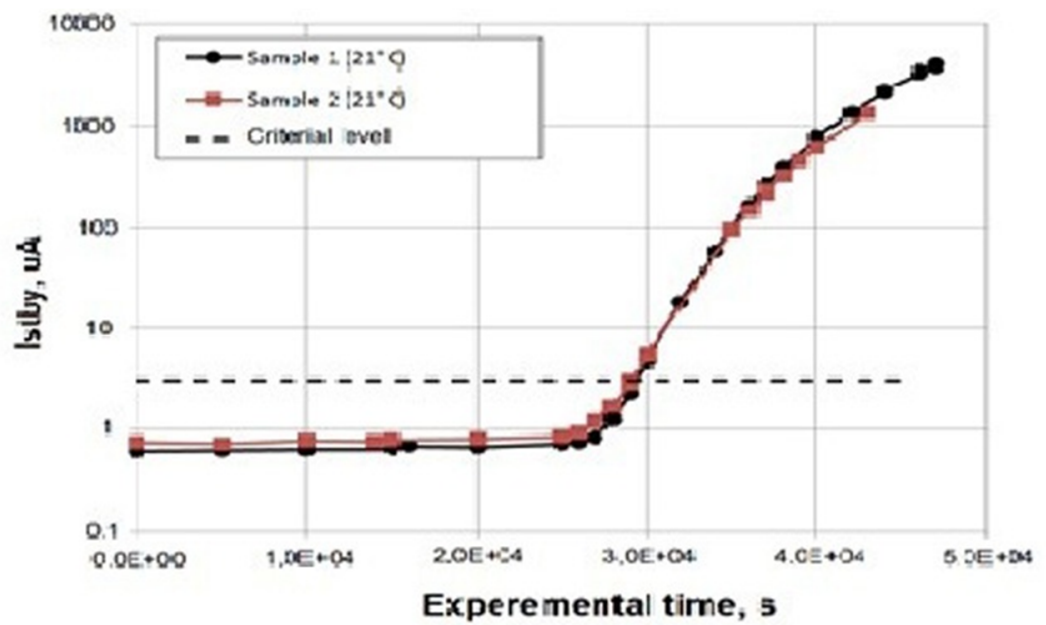

Figure 12. Sensors' standby current vs time. 


\section{Conclusions}

The digital temperature sensor DS1624S+ is one of the most used elements in different electrical systems. Two ways of communication with the temperature sensor DS1624S+ were designed. The developed systems can handle data from two independently connected temperature sensors in case of using built-in hardware I2C interface of the myRIO module and four independently connected temperature sensors in case of using NI myRIO FPGA based implementation of the I2C interface, measure the current consumption and the output voltage of the chips. The number of sensors is limited to the number of analog output channels. The experimental results are showed. Systems have been tested in the preparation of studies of temperature sensors in the AO "ENPO SPELS" and IEPE MEPhI.

\section{References}

[1] B. Karakozov, O. V. Korneev, P. V. Nekrasov, M. N. Sokolov, D. A. Zagryadsky, Proceedings of the European Conference on Radiation and its Effects on Components and Systems (RADECS), $6937407 \quad$ (2013) doi: 10.1109/RADECS.2013.6937407

[2] G. I. Zebrev, D. Y. Pavlov, V. S. Pershenkov, A.Y. Nikiforov, A. V. Sogoyan, D.V. Boychenko, V. N. Ulimov, V. V. Emelyanov, IEEE Transactions on Nuclear Science 53, 1981 (2006) doi: 10.1109/TNS.2006.877851

[3] G. V. Chukov, V.V. Elesin, G.N. Nazarova, A.Y. Nikiforov, D.V. Boychenko, V. A. Telets, A. G. Kuznetsov, K.M. Amburkin, IEEE Radiation Effects Data Workshop, 7004589 (2015) doi: 10.1109/REDW.2014.7004589

[4] O. A. Kalashnikov, V.V. Elesin, D.V. Gromov, 2014 24th International Crimean Conference Microwave and Telecommunication Technology Conference $\begin{array}{llll}\text { Proceedings } & \text { (CriMiCo } & \text { 2014), } & 6959666\end{array}$ doi: 10.1109/CRMICO.2014.6959666

[5] P. K. Skorobogatov, K. A. Epifantsev, O. A. Gerasimchuk, Russian Microelectronics 44, 40 (2015) doi: 10.1134/S1063739715010059

[6] P. K. Skorobogatov, A.Y. Nikiforov, O. B. Mavritsky, A. N. Egorov, A. V. Kirgizova, European Space Agency, (Special Publication) ESA SP (536), BP4 (2004)

[7] A. V. Grebenkina, D.V. Boychenko, A.Y. Nikiforov, V. A. Telets, V. V. Amelichev, V.A.Kharitonov, Proceedings of the International Conference on Microelectronics (ICM), 6842103 (2014) doi: 10.1109/MIEL.2014.6842103

[8] G. G. Davydov, A. S. Kolosova, L. N. Kessarinskiy, D.V. Boychenko, 2015 International Siberian Conference on Control and Communications (SIBCON 2015), 7147094 (2015) doi: 10.1109/SIBCON.2015.7147094

[9] N. E. Aristova, A. Y. Borisov, A. I. Tararaksin, L. N. Kessarinskiy, A. V. Yanenko, 2015 International Siberian Conference on Control and Communications (SIBCON 2015), 7146984 (2015) doi: 10.1109/SIBCON.2015.7146984

[10] I. O. Loskutov, A. B. Karakozov, P.V. Nekrasov, A.Y. Nikiforov, 2015 International Siberian Conference on Control and Communications (SIBCON 2015), 7147128 (2015) doi: 10.1109/SIBCON.2015.7147128

[11] I.I.Shvetsov-Shilovskiy, P.V. Nekrasov, A. V. Ulanova, A. A.Smolin, A. V. Sogoyan, 2015 International Siberian Conference on Control and Communications (SIBCON 2015), 7147282 (2015) doi: 10.1109/SIBCON.2015.7147282

[12] DS1624 Digital Thermometer and Memory Data Sheet URL: http://www.mouser.com/ds/2/256/DS1624-27266.pdf 
[13] NI myRIO-1900 user guide and specifications URL:http://www.ni.com/pdf/manuals/376047a.pdf

[14] V.A.Marfin, P.V. Nekrasov, O. A. Kalashnikov, K. A. Kagirina, 2015 International Siberian Conference on Control and Communications (SIBCON 2015), 7147142 (2015)

[15] D. V. Bobrovskii, O. A. Kalashnikov, P. V. Nekrasov, Russian Microelectronics 41, 226 (2012) doi: 10.1134/S1063739712040038 